\title{
Roll Angular Velocity Real-time Tracking Algorithm Based on Geomagnetic Information with Frequency-locked Loop
}

\author{
Zhen Wang ${ }^{\mathrm{a}}$, Fengqi Gao, Min Gao, Zhicai Lu \\ Shijiazhuang Mechanical Engineering College, Shijiazhuang 050003, China \\ awangzh822@sina.com
}

\begin{abstract}
A new rolling rate tracking method based on frequency-locked loop (FLL) was present in order to solve problems by low price, exact measurement and in-time correction mean for rocket projectile. The geomagnetic field component was firstly measured by magneto-resistive sensor and the frequency of signal was tracked by FLL. The geomagnetic field component regularity was analyzed in range of rocket projectile, which was changed by sine principle according to the trajectory character. The tracking algorithm of FLL was introduced in detail. In order to validate verification of the algorithm, the simulation model was build up by Matlab/Simulink. The simulation results showed that the algorithm could exactly track the frequency of the Chirp signal source from $5 \mathrm{~Hz}$ to $18 \mathrm{~Hz}$. In further, the tracking algorithm was implied on sampling geomagnetic field component data of rocket projectile. The experiment results indicated that it could satisfy the missile-borne controller need of low price and high accuracy.
\end{abstract}

Keywords-rocket projectile, frequency tracking, FLL, rolling frequency, Geomagnetic Information.

\section{INTRODUCTION}

United States, Israel, Germany and other countries have equipped with PGK trajectory correction fuze, ACLLAR160 represented trajectory correction weaponry, the use of "satellite navigation + geomagnetism" ballistic measurement technology system, the difference is mainly reflected in the implementation of the program adopted by agencies pathway can be divided into sub-caliber Canard, pulse engine, single-channel control scheme. There is a time delay between command and execution agency action amendment, the need for roll angle due to the delay caused by the implementing agencies for pre-judgment. There is a time delay between command and execution agency action correction, so we need to predict the roll angle. The idea of solving the time delay problem is by setting the delay time and measuring the roll rate to achieve a delay line compensation, thereby executing agency action directive issued in advance. Roll Attitude Measurement become one of the bottleneck problem of guidance ammunition trajectory correction efficiency.

Currently, the main measure of projectile roll attitude gyro and magnetoresistive sensors. When applied to a rotating gyroscope device rocket attitude measurement, there is a zero drift and over-range problems. We usually use isolation rotation measures measures or install anti-spin platform to measure projectile attitude. But these solutions are expensive, and not conducive to reduce the cost of the army's weapons and equipment. Magnetoresistive sensor having many advantages such as cheap, no accumulation of errors, anti-high overload, easy to mass production. This is the ideal device for Army equipment. Now, the technology of using magnetoresistive sensor to measure projectile roll angle is relatively mature, but measuring projectile roll rate is lagging behind. How to use magnetoresistive sensors simultaneously to measure the roll angle and angular velocity of projectile, is one of the key technologies to achieve cost reduction guided rocket projectile.

This paper presents a PLL-based low-cost roll rate real-time tracking is algorithm. Two-axis magnetoresistive sensor, which is mutually orthogonal in the transverse plane projectile, measures the component of the geomagnetic field and track the frequency change, enabling tracking and measurement of the angular velocity of roll. First, according to the law of rockets kinematics and Earth's magnetic field characteristics within the effective range. Secondly, we have established a roll angular velocity tracking model based PLL. According to the characteristics of the rocket roll rate changes, we simulated the tracking algorithm. Finally, the roll rate tracking algorithm is applied to the measured magnetic data. Using roll angle information based on a priori projectile, it need to pre-loaded rocket roll rate before the scheduled launch and use tracking algorithm to track and measure the angular velocity of roll.

\section{Roll Angular Velocity Tracking PRinciple BASED ON THE GEOMAGNETIC FIELD}

An easy way to comply with the conference paper formatting requirements is to use this document as a template and simply type your text into it.

Variation of geomagnetic field component in the projectile cross-section. In rocket flight, measuring the earth's magnetic field components by magnetoresistive sensor can achieve roll angular velocity tracking. Earth's magnetic field refers to the surface of the earth or the earth's space magnetic field, on the ground magnetic 
induction intensity is about 500 mGauss on average. Earth's magnetic field is composed of changing magnetic field and steady magnetic field changes, the former accounts for about $2 \sim 4 \%$ of total magnetic field. A certain type of rocket maximum range is about $30 \mathrm{~km}$. In this range, the magnetic field vector can be thought to be relatively constant, the magnetic field strength is $\mathrm{H}$. We set up north, day and east geographic coordinate system (N coordinate system for short). The angle between the Earth's magnetic field vector and the ground plane is Magnetic dip, marked as I. The angle between Earth's magnetic field vector and the North is magnetic declination, marked as D. On the axis of a geographic coordinate system, a projected component of the geomagnetic field are $H_{N}, H_{E}, H_{D}$, as shown in Fig. 1.

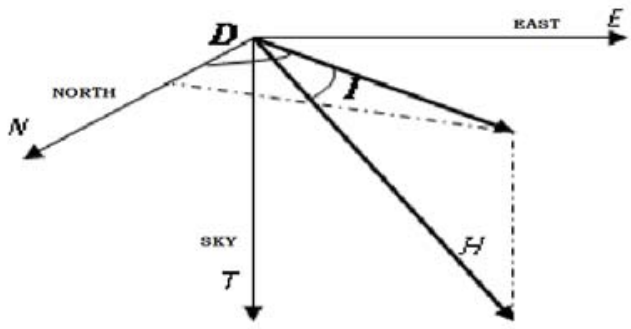

Figure 1. The relationship between the magnetic elements

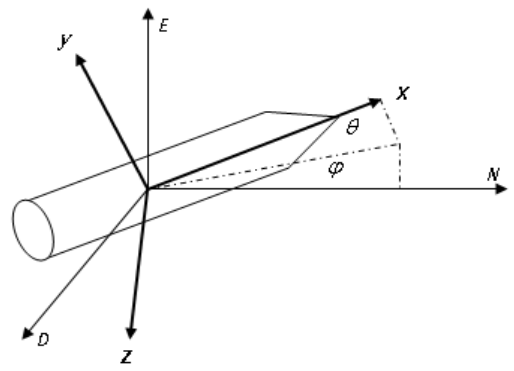

Figure 2. Coordinate system and their relationships

We establish a projectile coordinate system. ${ }^{o x_{1}}$ coordinate axis and the vertical axis of missile body are in the same direction, pointing to the head of the projectile direction is positive. In the vertical plane of symmetry within the elastomer, ${ }^{o y_{1}}$ axis is perpendicular to the ${ }^{o x_{1}}$ axis, pointing up. ${ }^{o z_{1}}$ axis is perpendicular to the ${ }^{o x_{1} y_{1}}$ axis. They constitute a right-handed coordinate system. The Angle between rocket vertical axis $\left({ }^{O X_{1}}\right)$ and the horizontal plane $(O X Z$ ) is pitching angle $\theta$. The angle between rocket vertical axis $\left({ }^{O X_{1}}\right)$ in the horizontal plane $O X z$ of projection is yaw angle $\psi$. The angle between axis ${ }^{o y_{1}}$ and vertical surface contained projectile vertical axis is roll angle $\gamma$. The relationship between the missile body coordinate system and the geographical coordinate system is shown in Fig. 2.
In the missile body coordinate system, the Earth's magnetic field component of the coordinate axes are ${ }^{H_{x}}$ 、 ${ }^{H_{y}}$ and $^{H_{z}}$.

$$
\begin{gathered}
{\left[\begin{array}{l}
H_{N} \\
H_{T} \\
H_{E}
\end{array}\right]=\left[\begin{array}{c}
H \cos I \cos D \\
H \sin I \\
H \cos I \sin D
\end{array}\right]} \\
{\left[\begin{array}{c}
H_{x} \\
H_{y} \\
H_{z}
\end{array}\right]=C_{n}^{1}\left[\begin{array}{l}
H_{N} \\
H_{T} \\
H_{E}
\end{array}\right]}
\end{gathered}
$$

Among them, $C_{n}^{1}$ is transformation matrix from the geographic coordinate system to the missile body coordinate.

$C_{n}^{1}=\left[\begin{array}{ccc}\cos \theta \cos \psi & \sin \theta & -\cos \theta \sin \psi \\ -\sin \theta \cos \psi \cos \gamma+\sin \psi \sin \gamma & \cos \theta \cos \gamma & \sin \theta \sin \psi \cos \gamma+\cos \psi \sin \gamma \\ \sin \theta \cos \psi \sin \gamma+\sin \psi \cos \gamma & -\cos \theta \sin \gamma & -\sin \theta \sin \psi \sin \gamma+\cos \psi \cos \gamma\end{array}\right]$

Then

$\left[\begin{array}{l}H_{y} \\ H_{2}\end{array}\right]\left[\left(-\sin \theta \cdot \cos \psi \cdot H_{N}+\cos \theta \cdot H_{T}+\sin \theta \cdot \sin \psi \cdot H_{E}\right) \cos \gamma+\left(\sin \psi \cdot H_{N}+\cos \psi \cdot H_{E}\right) \sin \gamma\right.$

$\left[\begin{array}{l}H_{y} \\ H_{z}\end{array}\right]=\left[\begin{array}{c}\left(-\sin \theta \cdot \cos \psi \cdot H_{N}+\cos \theta \cdot H_{T}+\sin \theta \cdot \sin \psi \cdot H_{E}\right) \cos \gamma+\left(\sin \psi \cdot H_{N}+\cos \psi \cdot H_{E}\right) \sin \gamma \\ \left(\sin \psi \cdot H_{N}+\cos \psi \cdot H_{E}\right) \cos \gamma+\left(\sin \theta \cdot \cos \psi \cdot H_{N}-\cos \theta \cdot H_{T}-\sin \theta \cdot \sin \psi \cdot H_{E}\right) \sin \gamma\end{array}\right]$

Assuming that:

$$
\begin{gathered}
K_{11}=-\sin \theta \cdot \cos \psi \cdot H_{N}+\cos \theta \cdot H_{T}+\sin \theta \cdot \sin \psi \cdot H_{E}, \\
K_{12}=\sin \psi \cdot H_{N}+\cos \psi \cdot H_{E} \\
K_{21}=\sin \psi \cdot H_{N}+\cos \psi \cdot H_{E}, \\
K_{22}=\sin \theta \cdot \cos \psi \cdot H_{N}-\cos \theta \cdot H_{T}-\sin \theta \cdot \sin \psi \cdot H_{E}
\end{gathered}
$$

Making these four coefficients into equation(4).

$\left[\begin{array}{c}H_{y} \\ H_{z}\end{array}\right]=\left[\begin{array}{l}K_{11} \cos \gamma+K_{12} \sin \gamma \\ K_{21} \cos \gamma+K_{22} \sin \gamma\end{array}\right]=\left[\begin{array}{l}A c_{y} \cos \left(\gamma+\gamma_{y}\right) \\ A c_{z} \cos \left(\gamma+\gamma_{z}\right)\end{array}\right]$

Among them

$$
\left[\begin{array}{c}
A c_{y} \\
A c_{z} \\
\gamma_{y} \\
\gamma_{z}
\end{array}\right]=\left[\begin{array}{l}
\sqrt{K_{11}{ }^{2}+K_{12}{ }^{2}} \\
\sqrt{K_{21}{ }^{2}+K_{22}{ }^{2}} \\
-\arctan \left(K_{12} / K_{11}\right) \\
-\arctan \left(K_{22} / K_{21}\right)
\end{array}\right]
$$

From rockets kinematic equations,

$$
\frac{d \gamma}{d t}=\omega_{x 4}-\omega_{y 4} \tan \theta
$$

$\omega_{x 4}$ and ${ }^{\omega_{y 4}}$ are subject to the projectile roll and yaw angular velocity in projectile coordinates. Integrating the angular velocity of roll on-time, we get the roll angle $\gamma=\omega_{x 4} t+\gamma_{0}$

The equation(7) into equation(5),

$$
\left[\begin{array}{l}
H_{y} \\
H_{z}
\end{array}\right]=\left[\begin{array}{c}
A c_{y} \cos \left(\omega_{x 4} t+\gamma_{y}^{\prime}\right) \\
A c_{z} \cos \left(\omega_{x 4} t+\gamma_{z}^{\prime}\right)
\end{array}\right]
$$

Analysis of ballistic parameters impacting on the Geomagnetic Field component. Considering the rocket engine parameters, aerodynamic parameters and test range weather data, we use 6-DOF motion equation for rocket 
exterior ballistic simulation. Launch angle is $42.57^{\circ}$,direction is north east $10^{\circ}$. Pitch angle $\theta$ and yaw angle $\psi$ are shown in Fig. 3.

As can be seen from Figure 3 ,rocket flight time of rise period is about $38 \mathrm{~s}$, pitching angle from $42.57^{\circ}$ dropped to $0^{\circ}$, pitching angle rate of about $1.1 \%$; rocket flight time of falling period is about 50s, pitching angle from $0^{\circ}$ dropped to $-63^{\circ}$, pitching angle rate of about $-1.2^{\circ} \%$. When the trajectory is determined, we can analysis of $\theta$ and $\psi$ impact on the changes in the geomagnetic field components.

The geomagnetic field strength of a test site is about $400 \mathrm{mGauss}$, Magnetic declination is $350^{\circ}$, magnetic dip is $-56^{\circ}$,launch angle is $42.57^{\circ}$, launch direction is north east $10^{\circ}$.According to $H_{N} 、 H_{E}, H_{D} 、 \theta$ and $\psi$, we can calculate the variation of $A c_{y}, A c_{z}, \gamma_{y}$ and $\gamma_{z}$ in equation(6). The result is shown in Fig. 4 - Fig. 6.

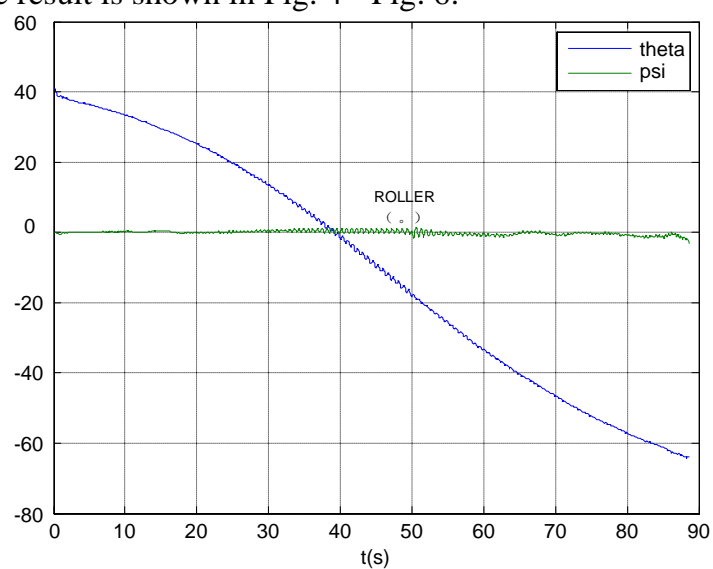

Figure 3. Time- Attitude Attitude

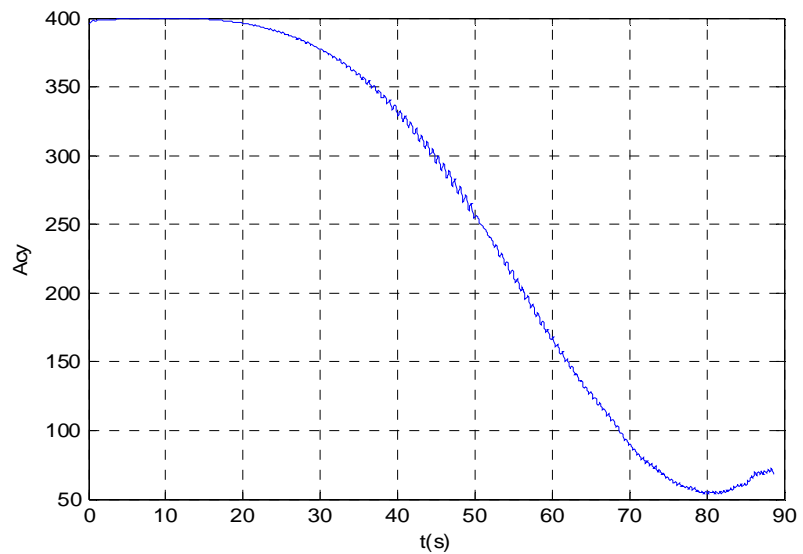

Figure 4. Time - Acy curve

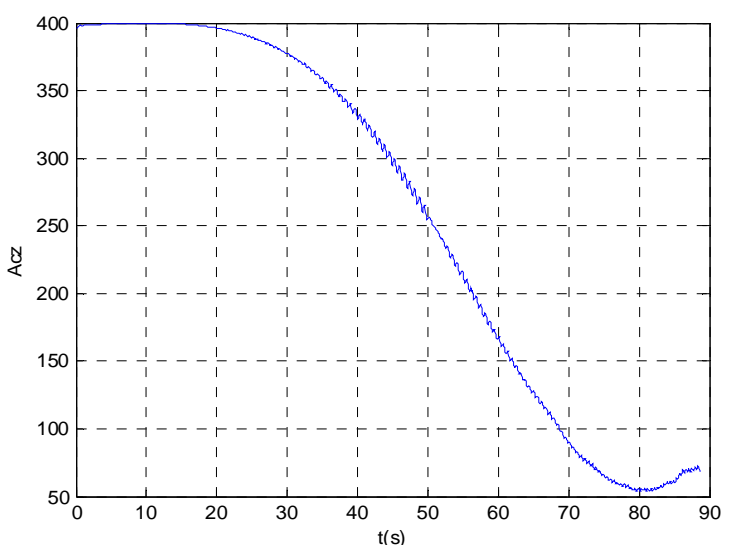

Figure 5. Time - Acz curve

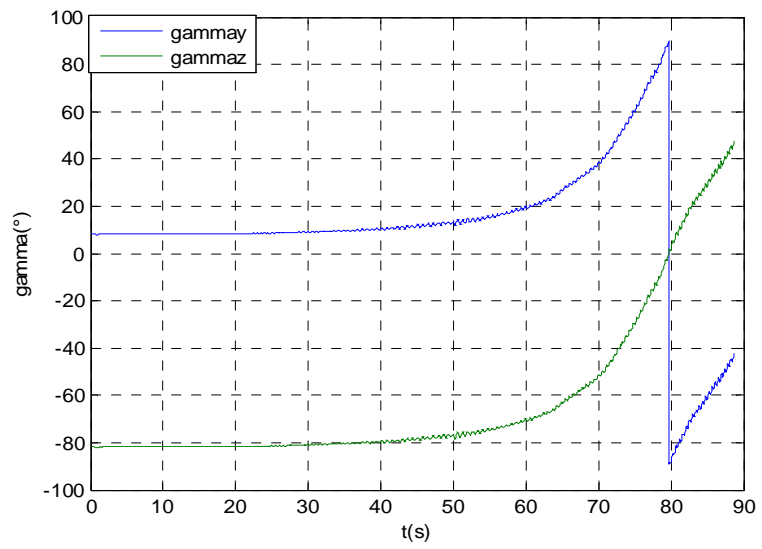

Figure 6. Time - Gamma curve

III. The Roll ANGUlar Velocity Tracking Algorithm BASEd ON FREQUENCY-LOCKED LOOP(FLL)

In the projectile transverse plane, we configure orthogonal magnetoresistive sensor and measure component of the geomagnetic field ${ }^{H_{y}}$ and ${ }^{H_{z}}$. Within a short period, it changes in line with the sine law. We use FLL on the geomagnetic field component signal tracking. Carrier tracking loop structure is shown in Fig. 7.

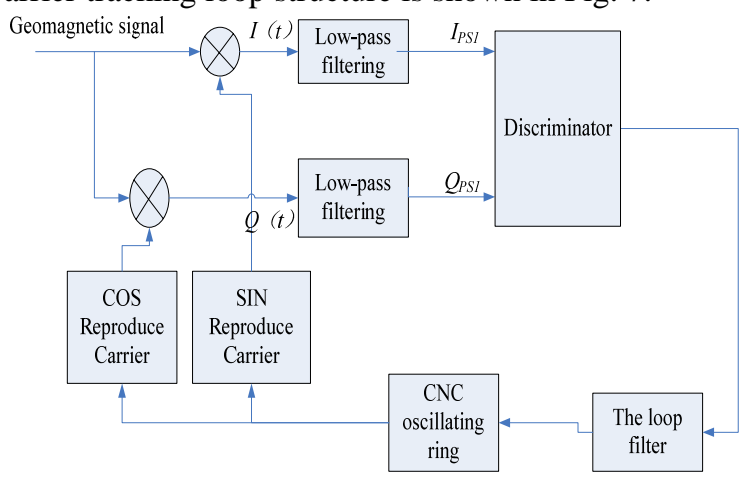

Figure 7. The principle diagram of carrier tracking loop 


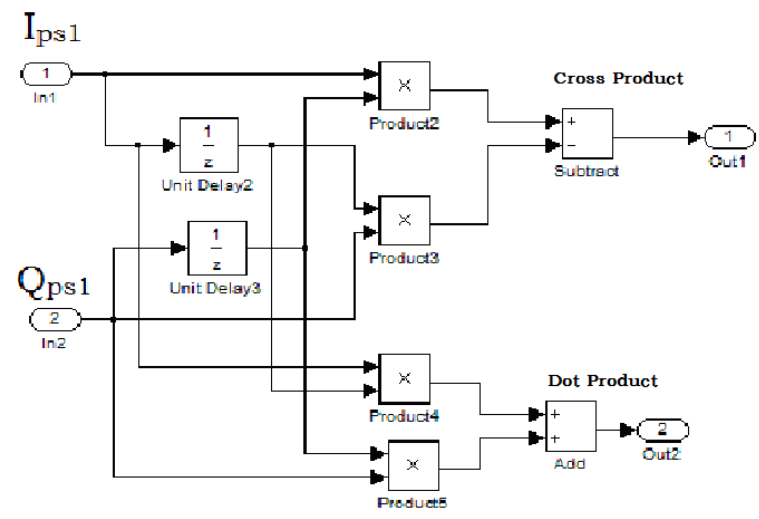

Figure 8. Phase structure diagram

According to the roll rate setted, we reproduce two carrier signals whose phase difference is $90^{\circ}$.The carrier signal frequency is same as setting angular rate. Geomagnetic signal is multiplied with two carrier signals respectively, then Low-pass filtering. The filtered signals are denoted as $I_{P S 1}$ and $Q_{P S 1} I_{P S 2}$ and ${ }^{Q_{P S 2}}$ are $I_{P S 1}$ and ${ }^{Q_{P S 1}}$ signals after being delayed. ${ }_{P S 1}$ and $Q_{P S 1}$ are processed after the phase detector to the loop filter. In the phase detector, it contains cross product and dot product, expressed by the following equations.

$$
\begin{gathered}
\text { Cross Product }=I_{P S 1} \times Q_{P S 2}-I_{P S 2} \times Q_{P S 1}=Q_{r s} \\
\text { Dot Product }=I_{P S 1} \times Q_{P S 2}+I_{P S 2} \times Q_{P S 1}=I_{r s}
\end{gathered}
$$

Phase structure is shown in Fig. 8.

After the Costas loop discriminator treatment, cross-product and dot product calculation output, then obtain the amount of change in the signal within a fixed time interval. By adjusting the bias signal digital oscillator frequency, it is succeeded in peeling the carrier frequence.

\section{SimUlATION AND TEST ANALYSIS}

Simulation analysis. To verify the validity of the angular rate tracking algorithm, we use Matlab/Simulink toolkit to model the track algorithm. The simulation model is shown in Fig. 9.

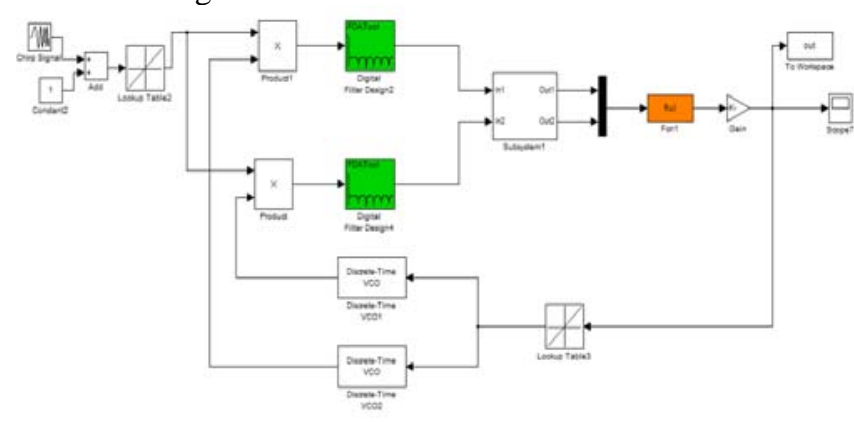

Figure 9. Roll angular velocity tracking algorithm model

Chirp Signal source is used as the excitation source, which frequency variation is $5-18 \mathrm{~Hz}$. Filter uses FIR low-pass filter, which cut-off frequency parameter is set to 0.01 , the sampling rate is set to $1 \mathrm{kHz}$. Discriminator uses $a \tan \left(Q_{r s} / I_{r s}\right)$ loop discriminator. Since the initial rocket speed can be pre-set, in the simulation of digital oscillator initial frequency is set to $5 \mathrm{~Hz}$. Simulink solver step size is set to a fixed formula, using ode4 (Runge-Kutta), a step size of $0.001 \mathrm{~s}$, simulation time $10 \mathrm{~s}$.

Frequency tracking error curve of simulation model is shown in Fig. 10.

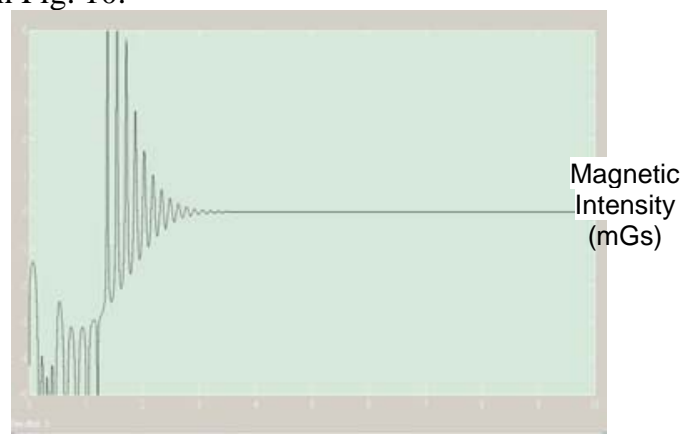

Figure 10. Frequency tracking error curve of simulation model

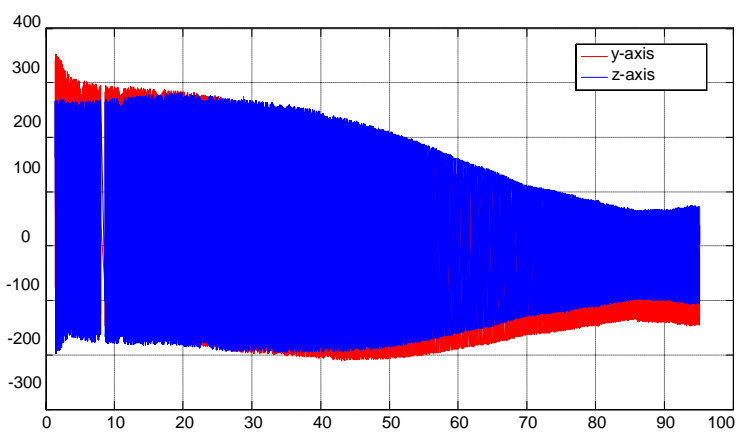

Figure 11. Magnetic sensor's output

As can be seen from Figure 11,In rocket flight, magnetoresistive sensor measurements of the geomagnetic field component in the range -300-400 mGauss. Increases with time, the intensity of the geomagnetic field components become smaller. In the last paragraph trajectory, ground magnetic field components in the range of -150-100 mGauss. This is because, in the rising phase of rocket flight, the pitch angle of the projectile decreases with increased range and positive, and the pitch angle is positive. In this stage the angle between the projectile axis and the magnetic field vector is gradually reduced. In the falling stage, projectile pitch angle is negative. The angle between the axis of the projectile pitch angle to the magnetic field vector decreases. In the trajectory tail section, the intensity of the geomagnetic field components have rise slightly, because the projectile pitch angle decreased. Component of the geomagnetic field data collection and Fig. 4, Fig. 5 simulation law is consistent. 
With the increase of the time and range, measurement of magnetic field components exist to zero drift. In the process of roll Angle computation, we need to be online calibration and compensation. This is due to the magnetoresistive sensor by environmental magnetic interference during operation. Zero drift have less effect on the missile roll angular velocity. 12.

The geomagnetic data of $30 \sim 30.2 \mathrm{~s}$ is shown in Fig.

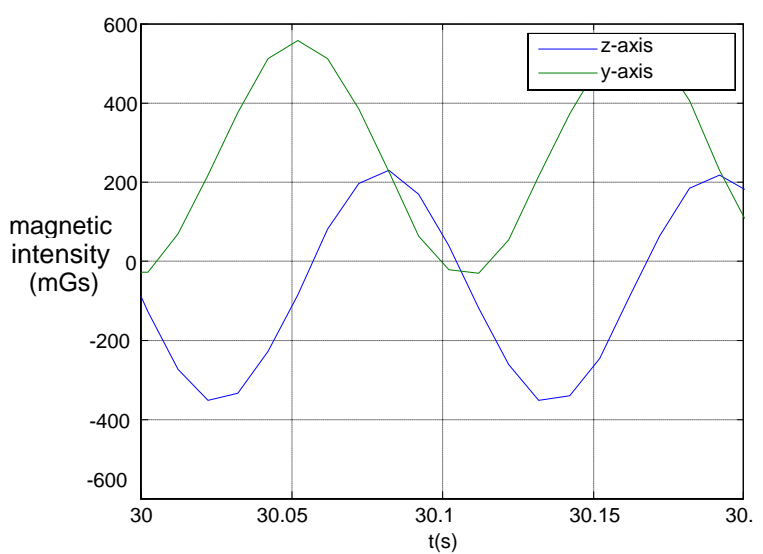

Figure 12. 30 30.2s magnetic sensor output

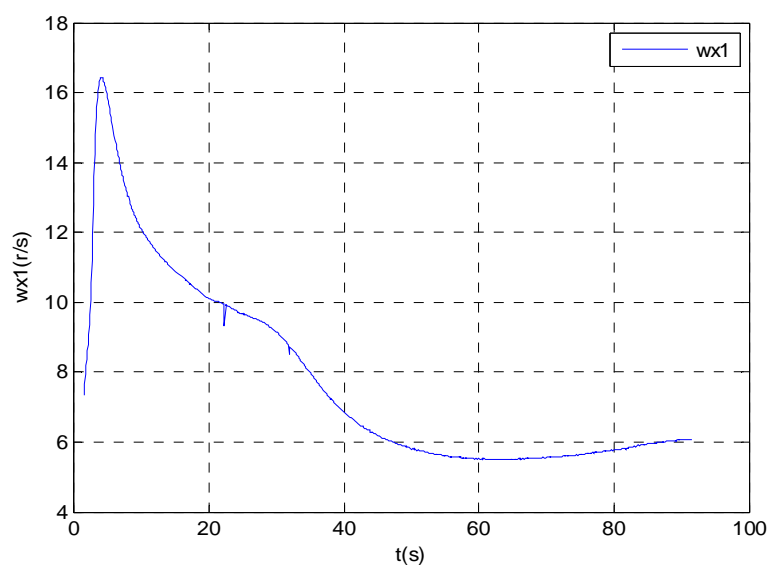

Figure 13. Tracking algorithm output results

In Fig. 13,roll rate had a sharp increase to a maximum in the initial segment, then roll rate gradually decreased to about 5 revolutions per second. In the late stage of trajectory, roll rate increased slightly. After the rocket launched, flight speed surge makes the roll torque increase. In the rising stage, the speed decreases. In the fall stage, with increasing flight speed rocket roll rate increase callback.

\section{CONCLUSION}

This paper proposes a method to track the rocket roll angular velocity. The method first sets parameters according to rocket leaving the launch tube velocity. Orthogonal magnetoresistive sensor configured in the projectile transverse plane measures component of the geomagnetic field, then we use a FLL to track the signal frequency of geomagnetic field components. Based on simulation, the method is feasible to meet the requirements of the on-rocket control system. In the rocket flight test, we made geomagnetic measuring device integrated into the on- rocket controllers. Tests verifies that our tracking method based on geomagnetic information works well. Geomagnetic field signal actually measured exexists zero drift, which is is related to the environment of the projectile and characteristic of the magnetoresistive sensor, but it does not affect tracking the rocket projectile roll angular velocity.

\section{REFERENCES}

[1] Philippe Wernert. Stability analysis for canard guided dual-spin stabilized projectiles [C]. AIAA Atmospheric Flight Mechanics.

[2] Kishore B Pamadi, Ernest J. Ohlmeyer. Assessment of a GPS Guided Spinning Projectile Using an Accelerometer-Only IMU[C]. AIAA Guidance, Navigation, and Control Conference and Exhibit 16 - 19 August 2004, Providence, Rhode Island:2-4.

[3] Mark Costello, Thanat Jitpraphai. Determining Angular Velocity and Angular Acceleration of Projectiles Using Triaxial Acceleration Measurements [J]. JOURNAL OF SPACECRAFT AND ROCKETS Vol. 39, No. 1, January-February 2002, 2010, 35(11):73-74.

[4] Zhenhua Wang, Yi Shen, Xiaolei Zhang. Attitude Sensor Fault Diagnosis based on Kalman Filter of Discrete-time Descriptor System[J]. Journal of Systems Engineering and Electronics Vol.23, No.6, December 2012:914-916.

[5] Davis, B. S. Using Low Cost MEMS Accelerometers and Gyroscopes as Strapdown IMUs on Rolling Projectiles[C]. Proceeding of the 1998 IEEE Position, Location, and Navigation Symposium, Vol. 1, No. 1, Inst. of Electrical and Electronics Engineers, New York, 1996:594-601.

[6] Zhao Handong, Cao Hongsong, Zhu Jizhi, Zhang,Huisuo. Method of Attitude Measurement with Magnetometer and Gyroscope[J].Jounal of North University of China Vol.31, No.6,2010:632-635.

[7] Gou Qiuxiong, Liu Mingxi, Li Hujun. Research of Terminal-Guide Mortar Bomb Roller Attitude Initial Alignment Technique Based on Magnetic Resistance Sensor[J]. Journal of Projectiles, Rockets, Missiles and Guidance Vol.23, No.3, Jun 2008:45-48.

[8] Shi Jinguang, Han Yan, Liu Shiping, Liu Meng. An Approach of Combination Measurement for Flight Attitude Angles of Guided Projectile[J]. Journal of Ballistics, Vol.23, No.3 Sep,2011:38-41.

[9] Wang Yong, Chen Jiabin, Liu Zhide, Song Chunlei. Study of Data Fusion Algorithms for Estimating Projectile Attitude[J]. Transactions of Beijing Institute of Technology, Vol.31, No.5, May 2011:534-536.

[10] FAN Jianpeng, FAN Hongqi, Lu Zaiqi.Pose estimation algorithm based on high range resolution profile. Journal of Systems Engineering and Electronics Vol.34, No.12:2413-2416. 